\title{
Heart and en-bloc thymus transplantation in miniature swine
}

\author{
Douglas R. Johnston, MD, a,d Ashok Muniappan, MD, a,d Ruediger Hoerbelt, MD, a,d Dax A. Guenther, MD, ${ }^{a, d}$ \\ Tsuyoshi Shoji, MD, PhD, ${ }^{\text {a,d }}$ Stuart L. Houser, MD, ${ }^{a, c, d}$ David H. Sachs, MD, ${ }^{\text {a,d }}$ and Joren C. Madsen, MD, DPhil ${ }^{a, b, d}$
}

\begin{abstract}
From the Transplantation Biology Research Center, ${ }^{\mathrm{a}}$ the Division of Cardiac Surgery, ${ }^{\mathrm{b}}$ and the Department of Pathology, ${ }^{\mathrm{c}}$ Massachusetts General Hospital, and Harvard Medical School, ${ }^{\mathrm{d}}$ Boston, Mass.

This work was supported in part by grants from the National Heart, Lung, and Blood Institute (RO1 HL54211, PO1 HL18646, and RO1 HL67110) of the National Institutes of Health. D.R.J. was supported in part by a Resident Research Scholarship from the American College of Surgeons and a National Institutes of Health (NIH) NRSA Training Grant (F32HL74519-02). A.M. was supported in part by the American Society of Transplantation/Sangstat Basic Science Fellowship, the American Society of Transplant Surgeons Thoracic Fellowship, and an NIH NRSA Training Grant (F32HL074671-02).
\end{abstract}

Received for publication Nov 30, 2004; revisions received Feb 22, 2005; accepted for publication March 8, 2005.

Address for reprints: Joren C. Madsen, MD, DPhil, Department of Surgery, Massachusetts General Hospital, 55 Fruit St, Boston, MA 02114 (E-mail: madsen@ helix.mgh. harvard.edu).

J Thorac Cardiovasc Surg 2005;130:554-9

$0022-5223 / \$ 30.00$

Copyright (C) 2005 by The American Association for Thoracic Surgery

doi:10.1016/j.jtcvs.2005.03.036
Background: Donor-specific tolerance to organ allografts might be induced by cotransplantation of a sufficient amount of vascularized donor thymus. To facilitate donor thymus-induced cardiac allograft tolerance, we have developed a novel technique for heart and en-bloc thymus transplantation in swine.

Methods: Donor heart and en-bloc thymus grafts were prepared by a technique that preserves the entire arterial supply and venous drainage of the right thymic lobe. En-bloc grafts $(n=4)$ were transplanted heterotopically into the abdomens of major histocompatibility complex-matched miniature swine. Recipients received 12 days of cyclosporine intravenously. Grafts were monitored by palpation, electrocardiographic monitoring, and periodic open biopsy. Engraftment of the donor thymus was demonstrated by measuring the proportion of recipient-type thymocytes in the donor thymus with flow cytometry.

Results: All of the heart and en-bloc thymus grafts had normal cardiac contractility and immediate perfusion of the thymus. All en-bloc grafts were accepted for more than 200 days without significant acute cellular rejection or cardiac allograft vasculopathy. Thymic tissue of en-bloc grafts displayed normal architecture and supported thymopoiesis of recipient-type cells.

Conclusion: We have validated a new technique of donor thymus transplantation that could have utility in human heart transplantation.

$\mathrm{T}$ he thymus prevents autoimmunity through a variety of mechanisms, including the negative selection of autoreactive $\mathrm{T}$ lymphocytes and the creation of regulatory T lymphocytes. ${ }^{1}$ These thymic functions are also important in the establishment of robust donor-specific tolerance in the transplant setting. ${ }^{2}$ Indeed, transplantation of donor thymus tissue results in donor-specific tolerance capable of prolonging cardiac allograft survival. ${ }^{3}$ Previously, thymic transplantation techniques in large animals depended on either pretransplantation modification of the donor organ by implantation of thymic tissue to create a composite graft (thymoheart or thymokidney) or on microsurgical techniques to transplant a vascularized thymic lobe. ${ }^{4,5} \mathrm{We}$ sought to develop a clinically relevant technique to confer large amounts of donor thymus to the recipient of a cardiac allograft at the time of organ transplantation. To that end, we developed an en-bloc technique that permits the cotransplantation of the heart and immediately vascularized thymus. This report describes our novel technique.

\section{Methods}

\section{Animals}

Heart and en-bloc thymus grafts were procured from and transplanted between animals belonging to partially inbred, major histocompatibility complex (MHC)-defined lines of Massachusetts General Hospital (MGH) miniature swine bearing mismatches only in minor histocompatibility antigens. ${ }^{6}$ Recipients were chosen that were positive for a PAA or CD4 
allele discrepant with the donor's allele. The CD4 and PAA alleles are detectable with available monoclonal antibodies. ${ }^{7}$ All animal care and procedures were in compliance with the "Principles of Laboratory Animal Care" formulated by the National Society for Medical Research and the "Guide for the Care and Use of Laboratory Animals" (NRC 1996); all animal experiments were approved by the Massachusetts General Hospital Subcommittee on Research and Animal Care.

\section{Heart and En-bloc Thymus Harvest}

The pig has 2 prominent cervical thymic lobes in addition to the mediastinal thymic lobes. Thymic vascular anatomy is very variable. Anatomic studies in various mammalian species suggest that thymic arterial supply and venous drainage are not predictable and are based on multiple branches. ${ }^{8,9}$ In this experiment the right cervical and mediastinal thymic lobes were harvested en bloc with the heart, which allowed the preservation of all arterial and venous branches to the thymus (Figure 1). The donor was placed in the supine position, and a midline cervical incision and sternotomy were performed. The sternohyoid and sternothyroid muscles were divided at their sternal attachments and retracted cephalad. The right carotid artery and internal jugular vein were isolated at the level of the cricothyroid membrane. The axillary artery and vein were isolated over the first rib. The left carotid artery (which arises from the innominate artery in the pig) was isolated approximately $3 \mathrm{~cm}$ distal to its origin. The left innominate vein was ligated and divided at the confluence of the internal jugular and axillary veins. The azygos vein was ligated at its origin from the superior vena cava. The right internal thoracic artery and internal thoracic vein were ligated and divided approximately $3 \mathrm{~cm}$ from their origins and were mobilized with care to preserve fine branches to the mediastinal thymus. The inferior vena cava (IVC) was isolated. The donor was systemically heparinized (200 U/kg), and all isolated vessels were ligated. The IVC was ligated and divided, permitting the heart to decompress. Cardioplegic solution (Plegisol; Abbott Laboratories, North Chicago, Ill) was administered through a catheter placed either in the aortic root or the left carotid stump, with a crossclamp applied to the aorta distal to the origin of the innominate artery and proximal to the origin of the left axillary artery (the clavicle is absent in pigs). After the heart was arrested and cardioplegic solution was delivered for 5 minutes, the heart and en-bloc thymus graft were dissected free of remaining attachments, the pulmonary veins were divided, the pulmonary artery was divided at its bifurcation, and the aorta was divided distal to the crossclamp. An atrial septal defect was created if not already present, and the small left atrial defect was repaired with a running suture (6-0 Prolene; Ethicon Inc, Somerville, NJ). The pulmonary veins and IVC were ligated.

\section{Heterotopic Heart and En-bloc Thymus Transplantation}

The recipient was placed in the supine position and had 2 indwelling lines placed in the external jugular veins. A midline laparotomy was performed, and the infrarenal aorta and IVC were isolated. After systemic heparinization (200 U/kg), the donor pulmonary artery was anastomosed in end-to-side fashion to the IVC with a continuous 6-0 Prolene suture. Next the donor aorta was anastomosed end to side to the recipient aorta in similar fashion. Before abdominal

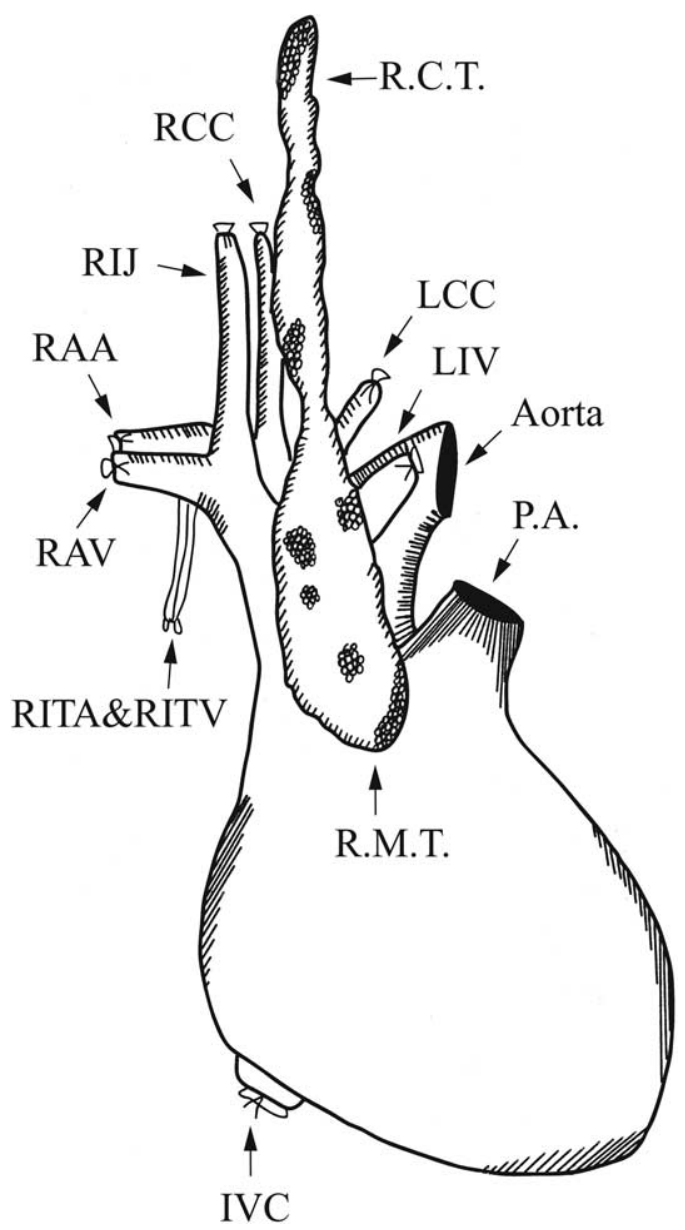

Figure 1. Miniature swine heart and en-bloc thymus graft. The vasculature of the en-bloc graft is depicted with respect to the right cervical and mediastinal thymic lobes. R.C.T., Right cervical thymus; R.M.T., right mediastinal thymus; $L C C$, left common carotid artery; $R C C$, right common carotid artery; $L I V$, left innominate vein; P.A., pulmonary artery; RITA, right internal thoracic artery; $R I T V$, right internal thoracic vein; $R A V$, right axillary vein; $R A A$, right axillary artery; $R I J$, right internal jugular vein; IVC, inferior vena cava.

closure, 2 iridium-tipped electrodes were implanted into the epicardium of each ventricle and brought out through the flank for long-term electrocardiographic monitoring.

\section{Postoperative Monitoring and Immunosuppression}

Graft viability was confirmed by daily abdominal palpation of the beating heart. ${ }^{10}$ Animals received cyclosporine (INN: ciclosporin) at a dose of 10 to $13 \mathrm{mg} \cdot \mathrm{kg}^{-1} \cdot \mathrm{d}^{-1}$ intravenously for 12 days, starting on the day of transplantation. Cyclosporine doses were adjusted to achieve serum trough levels of 400 to $800 \mathrm{ng} / \mathrm{mL}$. The heart and thymus were biopsied serially through a laparotomy and submitted for histologic analysis through hematoxylin and eosin stain and van Gieson elastin stain. 

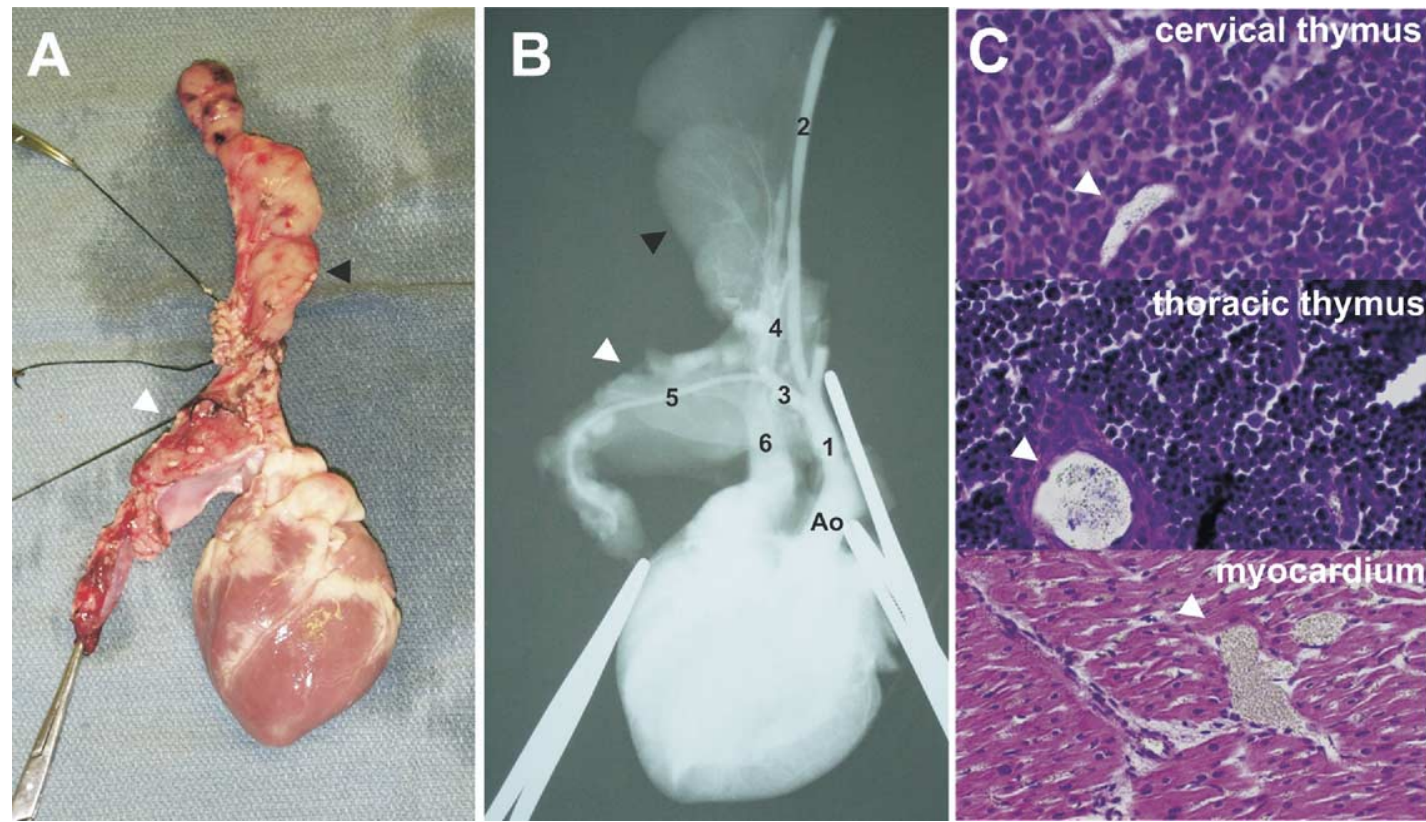

Figure 2. Arterial anatomy of heart and en-bloc thymus grafts. A, Gross specimen of en-bloc graft with display of the anterior view of the heart, right cervical thymus (black arrowhead), and right thoracic thymus (white arrowhead). B, Arterial angiography of en-bloc graft performed by means of infusion of barium into the aortic root (Ao). En-bloc dissection involves preservation of the innominate artery (1), right carotid artery (2), right axillary artery (3), thyrocervical trunk (4), and right internal thoracic artery (5), as well as fine thymic branches to the cervical (black arrowhead) and thoracic (white arrowhead) thymic lobes. Venous return is visualized in the superior vena cava (6). C, Hematoxylin and eosin-stained thymus and heart revealed the presence of barium in the small arteries, confirming perfusion.

\section{En-bloc Graft Angiography}

Barium was perfused into the aortic arch of one such heart and en-bloc thymus graft to visualize the thymic vasculature. Histologic examinations of sections of cervical thymus, mediastinal thymus, and myocardium were performed to detect barium in the small arteries.

\section{Assessment of Thymopoiesis}

Thymic allografts were assessed both histologically and by flow cytometry. Repopulation of the donor thymus by recipient thymo-

TABLE 1. Survival of MHC-matched heart and en-bloc thymus grafts

\begin{tabular}{lccc}
\hline Animal & Allograft & $\begin{array}{c}\text { Strain } \\
\text { combination }\end{array}$ & Survival (d)* \\
\hline 15168 & Heart and en-bloc thymus & $\mathrm{HH} \rightarrow \mathrm{HH}$ & $>274$ \\
15165 & Heart and en-bloc thymus & $\mathrm{HH} \rightarrow \mathrm{HH}$ & $>206$ \\
15228 & Heart and en-bloc thymus & $\mathrm{DD} \rightarrow \mathrm{DD}$ & $>277$ \\
15256 & Heart and en-bloc thymus & $\mathrm{DD} \rightarrow \mathrm{DD}$ & $>227$
\end{tabular}

$\overline{M H C}$, Major histocompatibility complex. *All animals were put to death with the heart beating. cytes was followed by flow cytometry and a recipient-specific antibody. Biopsies of the thymus were obtained serially at the time of heart biopsy. Thymic tissue was submitted for routine histologic study and was also processed by manual disruption and gradient separation (lymphocyte separation media; Organon Teknika, Durham, NC) to isolate thymocytes. Thymocytes were resuspended in flow cytometry medium (Hanks balanced salt solution, $0.1 \%$ bovine serum albumin, and $0.1 \% \mathrm{NaN}_{3}$ ) and stained with monoclonal antibodies specific for either the recipient $\mathrm{CD} 4$ allele (clone 74-12-4, IgG2b) or PAA allele (clone 1038H-10-9, IgM) and nonallelic CD1 (clone 76-7-4, IgG2a) for 30 minutes at $4{ }^{\circ} \mathrm{C} .{ }^{11}$ After washing, stained cells were analyzed on a FACScan (Becton Dickinson, San Jose, Calif).

\section{Results}

\section{Heart and En-bloc Thymus Transplantation}

A barium angiogram of a heart and en-bloc thymus graft (Figure 2, A) was performed, revealing the thymic vascular anatomy and preservation of fine thymic arterial branches (Figure 2, B). Histologic analysis revealed the presence of barium in the small arterial vessels of all sections of thymus examined, as well as the myocardium (Figure 2,C). On reperfusion of en-bloc grafts, the thymuses immediately 


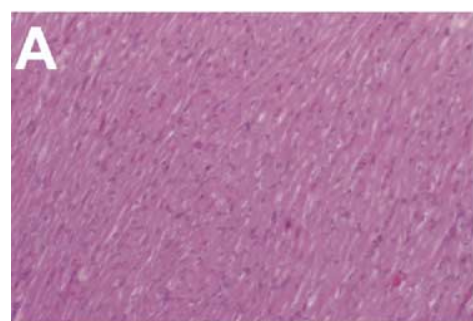

Heart, H\&E 100X

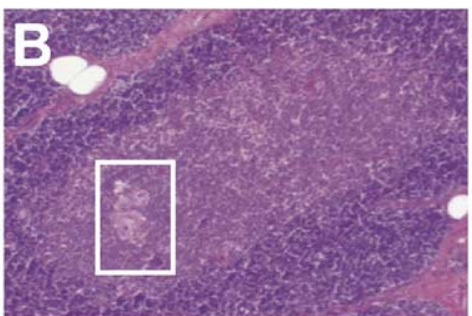

Thymus, H\&E 100X

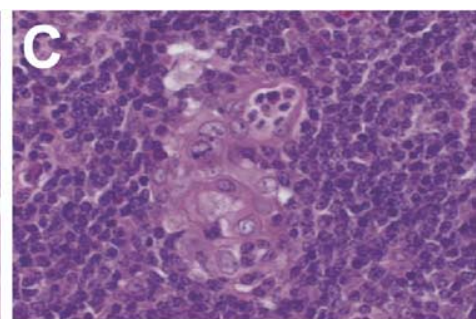

Thymus, H\&E 400X

Figure 3. Histology of heart and en-bloc thymus graft tolerated for more than 200 days. A, Hematoxylin and eosin (H\&E)-stained heart without evidence of acute rejection (International Society for Heart and Lung Transplantation 0/4). B, Hematoxylin and eosin-stained thymus revealing normal cortical and medullary architecture and a Hassals corpuscle (white box and C).

turned pink, reflecting immediate revascularization and adequate venous drainage.

\section{Survival}

Four consecutive heart and en-bloc thymus transplantations were performed between minor mismatched MGH miniature swine to test the long-term thympoietic function of the transplanted thymic grafts. After a short course of cyclosporine, all hearts survived indefinitely, and animals were put to death with the heart beating 200 days after transplantation (Table 1).

\section{Graft Histology}

Only 1 of the 4 heart grafts had any evidence of acute rejection (seen at 12 weeks, International Society for Heart and Lung Transplantation grade 2/4), which resolved before the next biopsy performed at 24 weeks after transplantation. There was no evidence of acute rejection at postmortem examination of all 4 hearts (Figure 3, A). Early biopsy specimens (4 weeks after transplantation) of all 4 thymic allografts revealed viable and normal-appearing thymic tissue on histopathology (Figure 3, B). Later biopsy specimens of each thymic graft showed evidence of focal thymic atrophy and fibrous replacement. However, some normalappearing thymic tissue was identified in all en-bloc grafts. Although allograft rejection cannot be absolutely ruled out, the focal histologic changes seen in the thymus likely represent a response to injury from the multiple biopsies. Thymic involution is observed in normally aging miniature swine as well, although this is typically a diffuse and generalized process, unlike what was observed in the thymic grafts.

Although this study was not designed to assess the effects of donor thymic transplantation on the host alloresponse, evaluation for chronic rejection of the cardiac allografts, as assessed by morphometric analysis, showed no significant incidence of cardiac allograft vasculopathy in all
4 en-bloc grafts (data not shown). Furthermore, these organs were maintained without significant acute or chronic rejection for more than 200 days without additional immunosuppression beyond the initial 12-day course of cyclosporine.

\section{Recipient Thymopoiesis in Grafts}

The function of transplanted thymic tissue was assessed by measuring recipient thymopoiesis in donor thymus grafts. Transplantations were performed between animals that differed in either CD4 or PAA alleles; recipient CD4 or PAA alleles were detectable with allele-specific monoclonal antibodies. Flow cytometry revealed increasing numbers of recipient-type thymocytes $\left(\mathrm{CD}^{+}\right)$in the donor thymus graft that reached proportions similar to those in the native thymus by 90 days after transplantation, as shown in 2 representative animals (Figure 4). The majority of cells isolated from the donor thymus were CD4/CD8 double positive and CD1 positive, confirming that they were thymocytes (data not shown). Furthermore, the normal proportion of recipient thymocytes in the donor thymus graft persisted for the duration of analysis (Figure 4), indicating that thymopoiesis was ongoing at the time animals were put to death. Parenthetically, peripheral blood mononuclear cells were isolated from recipients of en-bloc allografts, and macrochimerism was assessed by flow cytometry. Although low levels of donor-type lymphocytes were detected in the recipient's peripheral blood mononuclear cells initially after thymus transplantation, their levels quickly diminished with time, indicating that mixed chimerism was not established (data not shown).

\section{Discussion}

Although vascularized thymic transplantation has been performed in rodents by en-bloc harvest of the heart and thymus, ${ }^{12}$ there have been no descriptions of a clinically 


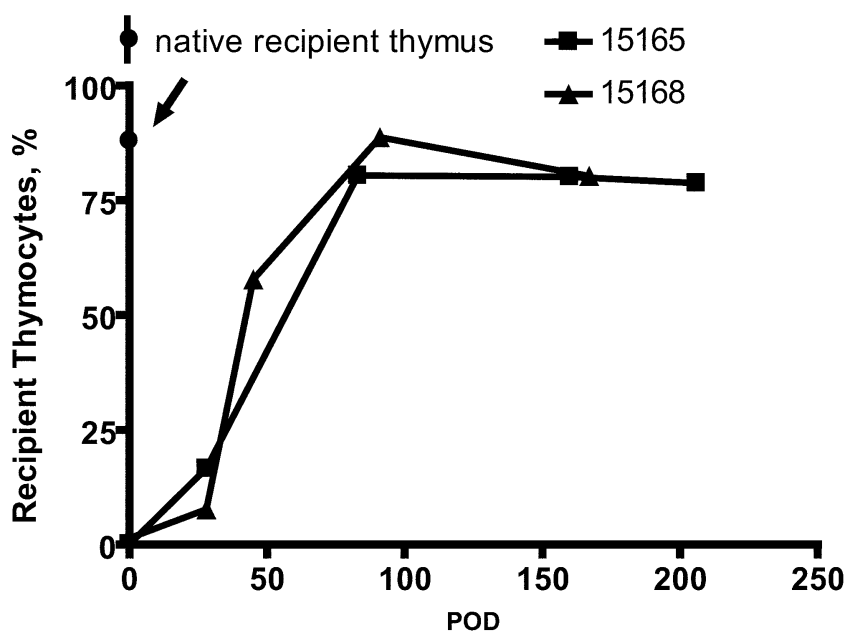

Figure 4. Recipient-type thymopoiesis in thymus grafts. Monoclonal antibody specific for either recipient CD4 or PAA allele was used to follow thymopoiesis of recipient cells in the donor thymus graft. Recipient thymocytes, $\mathrm{CD} 4^{+} \mathrm{CD}^{+}$or $\mathrm{PAA}^{+} \mathrm{CD} 1^{+}$double-positive cells, reached levels in the donor thymus comparable with levels seen in the native recipient thymus by postoperative day $\mathbf{1 0 0 .}$ Data from 2 animals are shown, which are representative.

applicable technique of heart and en-bloc thymus transplantation. We have established a new surgical technique that makes harvesting heart and en-bloc thymus grafts possible in large animals and human subjects. The heart and en-bloc thymus graft allows for immediate communication between the immune system of the host and the vascularized donor thymic tissue. Although tolerance has been achieved by grafting nonvascularized allogeneic thymic tissue into a variety of host sites in immunodeficient mice, ${ }^{13}$ efforts to cotransplant nonvascularized thymic tissue at the time of organ transplantation as a means of inducing tolerance in large animals have been unsuccessful. This might be due to rejection and loss of the thymic graft before the establishment of adequate vascularization. Indeed, the ischemic period in the first 1 to 3 weeks leads to temporary loss of the thymic graft structure, which is reconstituted over the next 5 to 7 weeks. ${ }^{14}$ During this period of revascularization, there is increased susceptibility to nonspecific graft loss and an increased potential for sensitization of the recipient.

We demonstrated that the heart and en-bloc thymus graft was capable of supporting thymopoiesis. It is hypothesized that thymopoiesis that occurs in the donor thymus will involve deletion of donor-specific $\mathrm{T}$ lymphocytes, generation of regulatory $\mathrm{T}$ lymphocytes, and development of an otherwise normal T-cell repertoire necessary for immunocompetence. ${ }^{5}$ Alternatively, thymic engraftment seen in these animals might be a consequence of tolerance induced by the short course of cyclosporine.
Future experiments will be performed to assess whether thymic cotransplantation can promote cardiac allograft tolerance across disparate MHC barriers. Across significant MHC barriers, we believe that stronger initial immunosuppression will be required to prevent acute rejection of the thymus and to permit its tolerogenic function. The en-bloc technique described here will also require validation in a nonhuman primate model because thymic anatomy in primates is slightly different. Our preliminary experiments suggest that primate thymic vasculature does not preclude application of the en-bloc technique.

Heart and en-bloc thymus transplantation supersedes our previous method for transplantation of vascularized thymus, which required a 2- to 3-month waiting period to allow autologous thymic tissue fragments to become engrafted under the donor heart epicardium. ${ }^{15}$ Not only does the en-bloc technique allow much more vascularized donor thymus to be conferred to the recipient at the time of organ transplantation, but it also eliminates the 2- to 3-month period of thymic engraftment. Thus this new technique makes the transfer of large amounts of vascularized donor thymus feasible in human recipients of cadaveric donor hearts, with minimal modification of current operative and immunosuppressive regimens. Because of the process of thymic involution, we believe that donor thymus cotransplantation will be most applicable to pediatric and adolescent transplant recipients. It also might have utility in cardiac xenotransplantation.

We thank Drs Leguern and Huang for their critical review of the manuscript, Ms Rebecca Hasse for her assistance in its preparation, and Dr Edirisinghe for the rendition in Figure 1.

\section{References}

1. Starr TK, Jameson SC, Hogquist KA. Positive and negative selection of T cells. Annu Rev Immunol. 2003;21:139-76.

2. Khan A, Tomita Y, Sykes M. Thymic dependence of loss of tolerance in mixed allogeneic bone marrow chimeras after depletion of donor antigen. Peripheral mechanisms do not contribute to maintenance of tolerance. Transplantation. 1996;62:380-7.

3. Menard MT, Schwarze ML, Allan JS, et al. Composite "thymoheart" transplantation improves cardiac allograft survival. Am J Transplant. 2004;4:79-86.

4. Yamada K, Vagefi PA, Utsugi R, et al. Thymic transplantation in miniature swine: III. Induction of tolerance by transplantation of composite thymokidneys across fully major histocompatibility complexmismatched barriers. Transplantation. 2003;76:530-6.

5. Kamano C, Vagefi PA, Kumagai N, et al. Vascularized thymic lobe transplantation in miniature swine: thymopoiesis and tolerance induction across fully MHC-mismatched barriers. Proc Natl Acad Sci U S A. 2004;101:3827-32.

6. Sachs DH, Leight G, Cone J, Schwarz S, Stuart L, Rosenberg S. Transplantation in miniature swine. I. Fixation of the major histocompatibility complex. Transplantation. 1976;22:559-67.

7. Fuchimoto Y, Huang C, Shimizu A, Seebach J, Arn S, Sachs DH. An allelic non-histocompatibility antigen with wide tissue distribution as a marker for chimerism in pigs. Tissue Antigens. 1999;54:43-52.

8. Di M, Argeme M, Brunet C, Coppens R, Bonnoit J. Macroscopic study of the adult thymus. Surg Radiol Anat. 1987;9:51-62.

9. Yamasaki M. Studies on the thyroid and thymic arteries of Japanese adults and fetuses. Anat Anz. 1989;169:213-21. 
10. Madsen JC, Sachs DH, Fallon JT, Weissman NJ. Cardiac allograft vasculopathy in partially inbred miniature swine. I. Time course, pathology, and dependence on immune mechanisms. J Thorac Cardiovasc Surg. 1996;111:1230-9.

11. Pescovitz MD, Lunney JK, Sachs DH. Preparation and characterization of monoclonal antibodies reactive with porcine PBL. J Immunol. 1984;133:368-75.

12. Ohuchi H, Madsen JC, Neragi-Miandoab S, Vlahakes GJ. A novel technique for en bloc, vascularized, composite thymic, and cardiac co-transplantation. Transplantation. 2002;74:403-5.
13. Waer M, Palathumpat V, Sobis H, Vandeputte M. Induction of transplantation tolerance in mice across major histocompatibility barrier by using allogeneic thymus transplantation and total lymphoid irradiation. J Immunol. 1990;145:499-504.

14. Haller GW, Esnaola N, Yamada K, et al. Thymic transplantation across an MHC class I barrier in swine. J Immunol. 1999;163: 3785-92.

15. Lambrigts D, Menard MT, Alexandre GPJ, et al. Creation of the "thymoheart" allograft: implantation of autologous thymus into the heart prior to procurement. Transplantation. 1998;66:810-4. 